\title{
Sums of Distinct Elements from a Fixed Set
}

\author{
By Torleiv Klove
}

\begin{abstract}
A sequence of natural numbers is complete if every large integer is a sum of distinct elements of the sequence. The greatest integer which is not such a sum is called the threshold of completeness. Richert developed a method to compute the threshold of completeness. We prove that Richert's method applies to a large class of complete sequences. Further, we consider in some detail these concepts for the sequences of powers (with fixed exponents) and give numerical results.
\end{abstract}

1. Let $M=\left\{m_{1}, m_{2}, \ldots\right\}$ be any increasing sequence of distinct natural numbers. $M$ is called complete if every sufficiently large integer may be expressed as a sum of distinct elements of $M$. If $M$ is complete, then there is a greatest integer which cannot be so expressed. This is called the threshold of completeness of $M$ and is denoted by $\theta(M)$.

A survey of papers on complete sequences is given by Graham [7]. The threshold of completeness has beell computed for a number of sequences by Sprague [13], Richert [11], [12], Makowski [10], Graham [6] , [7], Lin [9], and Dressler, Makowski and Parker [5]. Some of these values have been obtained independently by others (see [1] , [2] , [3] , [4] , [8]). The computations have been based on a theorem of Richert [11] or its underlying idea. An algorithm is given by Lin [9]. In this paper we give a partial answer to the question: For which complete sequences may Lin's algorithm be used to compute the threshold of completeness? In particular, we make a closer study of the threshold of completeness of the sequences of powers with fixed exponents.

2. We shall use the following notations:

$(a, b]$ denote the integers $n$ such that $a<n \leqslant b$, we call it an interval and $b-a$ is its length;

$$
\begin{aligned}
& M_{(k)}=\left\{m_{1}, m_{2}, \ldots, m_{k}\right\} \\
& N^{\alpha}=\left\{\left[n^{\alpha}\right] \mid n=1,2, \ldots\right\} \text { where }[x] \text { denote the greatest integer } \leqslant x ; \\
& N_{0}=\{0,1,2, \ldots\}
\end{aligned}
$$

we define a relation $\boldsymbol{A}$ between sequences of integers by: $P \boldsymbol{A} Q$ if each element of $P$ may be expressed as a sum of distinct elements of $Q$.

LEMMA 2.1. If $m_{i+1} \leqslant 2 m_{i}$ for $i>K$ and

$$
\left(a, a+m_{k+1}\right] \boldsymbol{E} M_{(k)}
$$

Received February 24, 1975.

AMS (MOS) subject classifications (1970). Primary 10A45, $10 \mathrm{~J} 99$.

Key words and phrases. Sums of distinct integers, sums of powers, complete sequences, threshold of completeness. 
for some $a \in N_{0}$ and some $k \geqslant K$, then

$$
\left(a, a+m_{l+1}\right] \boldsymbol{E} M_{(l)}
$$

for all $l \geqslant k$.

We prove (2.2) by induction on $l$. By (2.1) it is true for $l=k$. Suppose it is true for some $l \geqslant k$. Adding $m_{l+1}$ to each element of $\left(a, a+m_{l+1}\right]$ and merging the two intervals, we get $\left(a, a+2 m_{l+1}\right] \boldsymbol{A} \boldsymbol{M}(l+1)$. Since $m_{l+2} \leqslant 2 m_{l+1}$, this completes the induction.

THEOREM 2.1 (RICHERT [11]). If $m_{i+1} \leqslant 2 m_{i}$ for $i>K$ and $\left(a, a+m_{k+1}\right.$ ] E $M_{(k)}$ for some $a \in N_{0}$ and some $k \geqslant K$, then $M$ is complete and $\theta(M) \leqslant a$.

Proof. Let $b>a$. Since $m_{l} \rightarrow \infty$ for $l \rightarrow \infty, b \leqslant a+m_{l+1}$ for some $l$. By Lemma $2.1\{b\} \boldsymbol{E} M_{(l)}$. Hence $\{b\} \boldsymbol{E} \boldsymbol{M}$.

Theorem 2.1 provides a method to calculate $\theta(M)$. A discussion of an algorithm is given by Lin [9]. The crucial point is the existence of $a$ and $k$ such that (2.1) is satisfied. This will be further discussed in Lemma 2.2 and Theorem 2.3 below. If we want to compute $\theta_{n}=\theta\left(\left\{m_{n}, m_{n+1}, \ldots\right\}\right)$ for $n=1,2, \ldots, l$, the algorithm may be modified to yield all these values in one run (see Kl $\phi$ ve [8]). We give a sketch of this modified algorithm.

Algorithm $\theta$.

Step 1. $k:=l-1 ; N_{l-1}=\{0\}$;

Step 2. $k:=k+1 ; N_{k}=N_{k-1} \cup\left\{n+m_{k} \mid n \in N_{k-1}\right\}$;

Step 3. If $k<K$, then go to Step 2 .

Step 4. If $N_{k}$ does not contain an interval of length $\geqslant m_{k+1}$, then go to Step 2;

Step 5. $a:=$ least $x$ such that $\left(x, x+m_{k+1}\right] \subseteq N_{k}$;

Step 6. $k:=k+1$; if $m_{k}>$ a,then go to Step 8;

Step 7. $N_{k}:=N_{k-1} \cup\left\{n+m_{k} \mid n \in N_{k-1} \& n \leqslant a-m_{k}\right\}$;

$a:=$ least $x$ such that $(x, a+1] \subseteq N_{k}$; go to Step 6;

Step 8. $\theta_{l}:=a$;

Step 9. $k:=l$;

Step 10. If $k>1$, then $k:=k-1$, else stop.

Step 11. $N_{k}:=N_{k+1} \cup\left\{n+m_{k} \mid n \in N_{k+1} \& n \leqslant \theta_{k+1}-m_{k+1}\right\}$;

$\theta_{k}:=$ least $x$ such that $\left(x, \theta_{k+1}+1\right] \subseteq N_{k}$; go to Step 10.

The actual algorithm will depend on how we choose to represent the sets $N_{k}$.

Clearly, for $l=1$ the algorithm will give just $\theta(M)$.

The following theorem may be used to simplify the algorithm (this simplified algorithm will usually be less efficient, however).

THEOREM 2.2. If $m_{i+1} \leqslant 2 m_{i}$ for $i>K$ and

$$
\left(a, a+m_{k+1}\right] \boldsymbol{E} \boldsymbol{M}_{(k)}
$$

for some $a \in N_{0}$ and some $k \geqslant K$, then

$$
\left(\theta(M), \theta(M)+m_{l+1}\right] \boldsymbol{A} M_{(l)} \text { for some } l \geqslant K .
$$

Proof. Let $a^{*}=\min \{a \mid(2.3)$ is satisfied for some $k \geqslant K\}$. By Theorem $1, a^{*} \geqslant$ $\theta(M)$. Suppose $a^{*}>\theta(M)$. Then $a^{*}=\Sigma_{j} m_{i_{j}}$ where $i_{j} \leqslant l_{1}$, for some $l_{1}$. By assump- 
tion

$$
\left(a^{*}, a^{*}+m_{L+1}\right] \boldsymbol{A} M_{(L)}
$$

for some $L \geqslant K$. Let $l=\max \left(l_{1}, L\right)$. Then, by Lemma $2.1\left(a^{*}, a^{*}+m_{l+1}\right] \boldsymbol{E} M_{(l)}$. Further $\left\{a^{*}\right\} \boldsymbol{A} M_{(l)}$. Hence

$$
\left(a^{*}-1, a^{*}-1+m_{l+1}\right] \boldsymbol{E} M_{(l)}
$$

contradicting the definition of $a^{*}$.

We now turn to the question: For which complete sequences may the algorithm above be used to compute the threshold of completeness? A partial answer is given by the following lemma and Theorem 2.3 below.

LEMMA 2.2. If $M$ is complete and $m_{k+1} \leqslant 2 m_{k}-\theta(M)-1$ for $k \geqslant K$, let $L$ be the minimum $l \geqslant K$ such that $m_{l+1} \geqslant 1+\theta(M)+m_{k}$. Then for all $l \geqslant L$

$$
\left(\theta(M), \theta(M)+m_{l+1}\right] \boldsymbol{A E} M_{(l)} .
$$

Proof. Let $\theta=\theta(M)$. We may assume that $m_{K} \geqslant \theta-2$ (otherwise we just increase $K)$. First, we prove by induction on $n$ that

$$
\left(\theta, m_{n+1}-1\right] \cup\left(\theta+m_{K}, 2 m_{n}-1\right] \boldsymbol{A E} M_{(n)}
$$

for $n \geqslant K$. By assumption $\left(\theta, m_{n+1}-1\right] \boldsymbol{E} M$ and since $m_{k}$ for $k \geqslant n+1$ may never occur as a summand, we get

$$
\left(\theta, m_{n+1}-1\right] \boldsymbol{A} M_{(n)}
$$

for all $n$. In particular, (2.5) is true for $n=K-1$. Adding $m_{K}$ to each element,we get $\left(\theta+m_{K}, 2 m_{K}-1\right] \boldsymbol{E} M_{(K)}$, and so (2.4) is true for $n=K$. Suppose it is true for some $n \geqslant K$. In particular,

$$
\left(\theta+m_{K}, 2 m_{n}-1\right] \boldsymbol{A} M_{(n)} .
$$

Adding $m_{n+1}$ to each element of the set in (2.5), we get

$$
\left(\theta+m_{n+1}, 2 m_{n+1}-1\right] \boldsymbol{E} M_{(n+1)} \text {. }
$$

Since $\theta+m_{n+1}<2 m_{n},(2.5),(2.6)$, and (2.7) show that (2.4) is true for $n$ replaced by $n+1$ and the induction is complete. Now choose $l \geqslant K$ such that $m_{l+1} \geqslant \theta+1$ $+m_{K}$. Then $\left(\theta, 2 m_{l}-1\right] \boldsymbol{E} M_{(l)}$. Finally,

$$
2 m_{l}-1=\theta+\left(2 m_{l}-1-\theta\right) \geqslant \theta+m_{l+1}
$$

and the lemma is proved.

THEOREM 2.3. Let $M$ be complete. If, for some $\epsilon>0, m_{l+1} \leqslant(2-\epsilon) m_{l}$ for all $l \gg 0$, then $\theta(M)$ may be computed by algorithm $\theta$.

Proof. Choose $k>K$ such that $\epsilon m_{k} \geqslant \theta(M)+1$. Then

$$
m_{k+1} \leqslant 2 m_{k}-\epsilon m_{k} \leqslant 2 m_{k}-\theta(M)-1 \text {. }
$$

By Lemma 2.2 there exists an $l$ such that

$$
\left(\theta(M), \theta(M)+m_{l+1}\right] \boldsymbol{E} M_{(l)}
$$

and algorithm $\theta$ applies. 
We now give a trivial lemma which may be used to find a lower bound for $\theta(M)$. LEMMA 2.3. If $M$ is complete, then

$$
\theta(M) \geqslant \max _{n}\left(m_{n+1}-2^{n}-1\right) .
$$

Proof. The number of distinct sums of distinct elements of $M_{(n)}$ is $\leqslant 2^{n}$. Hence, if $2^{n}+1 \leqslant m_{n+1}$, then there is at least one integer in $\left[m_{n+1}-2^{n}-1, m_{n+1}\right)$ which cannot be expressed as a sum of distinct elements of $M$. Hence $\theta(M) \geqslant m_{n+1}$ $-2^{n}-1$.

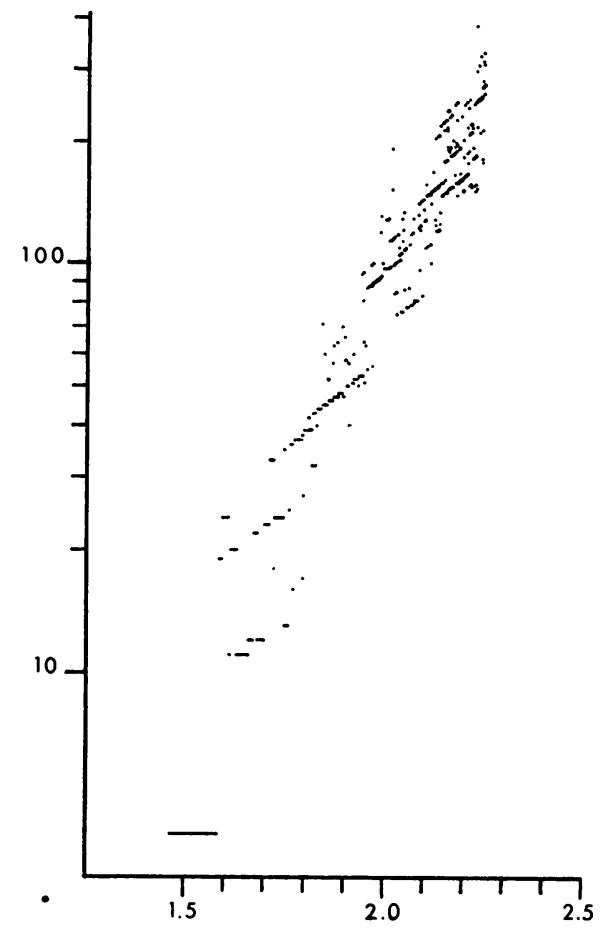

FIGURE 1. $t(\alpha)$ for $1.50 \leqslant \alpha \leqslant 2.25$.

3. Sprague [13], [14] proved that $N^{\alpha}$ is complete for $\alpha=2,3, \ldots$ and found that $\theta\left(N^{2}\right)=128$. Since $N^{n}$ is a subsequence of $N^{m / m}, N^{\alpha}$ is complete for all rational $\alpha$. Graham [6] proved that $\theta\left(N^{3}\right)=12758$ and Lin [9] that $\theta\left(N^{4}\right)=5134240$. Let $t(\alpha)=\theta\left(N^{\alpha}\right)$. The rest of this paper is concerned with $t(\alpha)$.

THEOREM 3.1. If $N^{\beta}$ is complete, then there exists $a \beta^{\prime}>\beta$ such that $t(\alpha)$ is constant on $\left[\beta, \beta^{\prime}\right)$.

Note that $\left[\beta, \beta^{\prime}\right)$ denotes an interval in the ordinary sense.

Proof. Clearly $\left[(k+1)^{\beta}\right]<2\left[k^{\beta}\right]-t(\beta)-1$ for $k \gg 0$. Hence, by Lemma 2.2 , there exists an $l$ such that

$$
\left(t(\beta), t(\beta)+\left[(l+1)^{\beta}\right]\right] A E N_{(l)}^{\beta} .
$$

By Lemma 2.1 we may assume that

$$
\left[l^{\beta}\right]>t(\beta) .
$$


TABLE 1

Values of $t(0.1 \beta+0.01 \gamma)$

\begin{tabular}{r|rrrrr}
$\beta$ & 0 & 2 & 4 & 6 & 8 \\
\cline { 2 - 6 } 15 & 0 & 4 & 4 & 4 & 4 \\
16 & 4 & 20 & 11 & 11 & 22 \\
17 & 24 & 33 & 24 & 13 & 37 \\
18 & 39 & 43 & 44 & 46 & 64 \\
19 & 58 & 51 & 94 & 88 & 91 \\
20 & 128 & 115 & 76 & 111 & 81 \\
21 & 127 & 169 & 147 & 193 & 159 \\
22 & 166 & 183 & 259 & 221 & 294 \\
23 & 306 & 310 & 306 & 517 & 409 \\
24 & 530 & 646 & 728 & 493 & 1095 \\
25 & 724 & 702 & 975 & 798 & 892 \\
26 & 1039 & 1180 & 1583 & 1324 & 1671 \\
27 & 1702 & 1741 & 1791 & 1925 & 2286 \\
28 & 1987 & 2873 & 2671 & 3909 & 4711 \\
29 & 3821 & 4882 & 4312 & 4866 & 5814 \\
30 & 12758 & 7110 & 6206 & 8895 & 8536 \\
31 & 13399 & 10594 & 11530 & 11008 & 16458 \\
32 & 11335 & 18114 & 18654 & 18165 & 21637 \\
33 & 21090 & 24770 & 23807 & 28420 & 29178 \\
34 & 32666 & 40701 & 40018 & 46056 & 49473 \\
35 & 54714 & 54869 & 56832 & 65719 & 86669 \\
36 & 74648 & 95679 & 88685 & 94399 & 109647 \\
37 & 132511 & 126425 & 158215 & 177256 & 174059 \\
38 & 206162 & 213823 & 240047 & 247208 & 286548 \\
39 & 324446 & 331169 & 365194 & 395838 & 403710 \\
40 & 5134240 & 483960 & 635701 & 610633 & 726860 \\
41 & 742867 & 831254 & 812774 & 984068 & 981046
\end{tabular}

Choose $\beta^{\prime}>\beta$ such that $\left[n^{\alpha}\right]$ is constant on $\left[\beta, \beta^{\prime}\right)$ as a function of $\alpha$ for $n=1,2$, ..., $l+1$, e.g.

$$
\beta^{\prime}=\min \left\{\log \left(\left[n^{\beta}\right]+1\right) / \log n \mid 1<n \leqslant l+1\right\} .
$$

Then, for all $\alpha \in\left[\beta, \beta^{\prime}\right)$,

$$
\left(t(\beta), t(\beta)+\left[(l+1)^{\alpha}\right]\right] A E N_{(l)}^{\alpha} .
$$

By Theorem $2.1 t(\alpha) \leqslant t(\beta)$. Suppose $t(\alpha)<t(\beta)$ for some $\alpha \in\left[\beta, \beta^{\prime}\right)$. Then

$$
t(\beta)=\sum_{j}\left[n_{j}^{\alpha}\right]=\sum_{j}\left[n_{j}^{\beta}\right]
$$

since $n_{j}<l$ for all $j\left(\left[n_{j}^{\alpha}\right] \leqslant t(\beta)<\left[l^{\beta}\right] \leqslant\left[l^{\alpha}\right]\right)$, a contradiction. Hence $t(\alpha)=t(\beta)$ for all $\alpha \in\left[\beta, \beta^{\prime}\right)$. 
We see that, given $\beta$, we may compute a $\beta^{\prime}$ satisfying the theorem as follows: Using algorithm $\theta$, we compute an $l$ satisfying (3.1) and (3.2). Then a $\beta^{\prime}$ is given by (3.3). Further, we may replace $\beta$ by $\beta^{\prime}$ and compute a new interval. Using this algorithm, we computed $t(\alpha)$ for $1 \leqslant \alpha \leqslant \log 686 / \log 15 \approx 2.412$. In particular,

$$
\begin{array}{ll}
t(\alpha)=0 & \text { for } 1 \leqslant \alpha<\log 5 / \log 3 \approx 1.465 \\
t(\alpha)=4 & \text { for } \log 5 / \log 3 \leqslant \alpha<\log 3 / \log 2 \approx 1.585
\end{array}
$$

A plot of $t(\alpha)$ versus $\alpha$ for $1.50 \leqslant \alpha \leqslant 2.25$ is given in Fig. 1 .

We have computed $t(\alpha)$ for $\alpha=1.50$ (0.02) 4.18 using algorithm $\theta$. The values are given in Table 1. (The value for $t(4)$ is taken from Lin [9] and has not been recomputed.) It is striking that the value of $t(\alpha)$ at $\alpha=4$ is much greater than the surrounding values. The referee suggests that this is probably due to the fact that $x^{4} \equiv 0,1(\bmod 16)$.

Acknowledgment. Thanks to the referee, the exposition has been improved in some places. In particular, Lemma 2.3 has been sharpened. Further, the referee has noticed that all the results in this paper are valid for nondecreasing sequences $M$. If $m_{k} \rightarrow \infty$ nothing needs to be changed. If $m_{k}$ is constant for $k \geqslant K$, consider the sequence $M^{\prime}=\left(m_{1}, m_{2}, \ldots, m_{K-1}, m_{K}, 2 m_{K}, 3 m_{K}, \ldots\right)$.

Matematisk Institutt, avd. A

Universitetet i Bergen

N-5014 Bergen, Norway

1. R. E. DRESSLER, "A stronger Bertrand's postulate with an application to partitions," Proc. Amer. Math. Soc., v. 33, 1972, pp. 226-228. MR 45 \#1828.

2. R. E. DRESSLER, "Addendum to: A stronger Bertrand's postulate with an application to partitions," Proc. Amer. Math. Soc., v. 38, 1973, p. 667. MR 46 \#8947.

3. R. E. DRESSLER, "Sums of distinct primes," Nordisk Mat. Tidskr., v. 21, 1973, pp. 31 32. MR 48 \#3851.

4. R. E. DRESSLER \& T. PARKER, “12,758,” Math. Comp., v. 28, 1974, pp. 313-314. MR 48 \#5994.

5. R. E. DRESSLER, A. MAKOWSKI \& T. PARKER, "Sums of distinct primes from congruence classes modulo 12," Math. Comp., v. 28, 1974, pp. 651-652.

6. R. L. GRAHAM, "Complete sequences of polynomial values," Duke Math. J., v. 31, 1964, pp. 275-285. MR 29 \#63.

7. R. L. GRAHAM, "On sums of integers taken from a fixed sequence," Proceedings of the Washington State University Conference on Number Theory (Washington State Univ., Pullman, Wash., 24-27 March, 1971), Dept. of Math., Pi Mu Epsilon, Washington State Univ., Pullman, Wash., 1971, pp. 22-40. MR 47 \#4904.

8. T. KL $\emptyset$ VE, "Sums of distinct primes," Nordisk Mat. Tidskr., v. 21, 1973, pp. 138-140.

9. S. LIN, "Computer experiments on sequences which form integral bases," Computa-

tional Problems in Abstract Algebra (Proc. Conf., Oxford, 1967), Pergamon, Oxford, 1970, pp. 365370. MR 41 \#169.

10. A. MAKOWSKI, "Partitions into unequal primes," Bull. Acad. Polon. Sci. Sér. Math. Astronom. Phys., v. 8, 1960, pp. 125-126. MR 22 \#7991.

11. H. E. RICHERT, "Über Zerlegungen in paarweise verschiedene Zahlen," Norsk Mat. Tidsskr., v. 31, 1949, pp. 120-122. MR 11, 646.

12. H. E. RICHERT, "Über Zerfällungen in ungleiche Primzahlen," Math. Z., v. 52, 1950, pp. 342-343. MR 11, 502.

13. R. SPRAGUE, “Über Zerlegungen in ungleiche Quadratzahlen," Math. Z., v. 51, 1948, pp. 289-290. MR 10, 283.

14. R. SPRAGUE, "Über Zerlegungen in $n$-te Potenzen mit lauter verschiedenen Grundzahlen," Math. Z., v. 51, 1948, pp. 466-468. MR 10, 514. 